\title{
Pseudomyxoma peritonei of appendiceal origin mimicking ovarian cancer - a case report with literature review
}

\author{
Stoyan Kostov ${ }^{1}$, Yavor Kornovski², Stanislav Slavchev², Yonka Ivanova², Deyan Dzhenkov³, \\ Angel Yordanov 4 , Svetoslava Slavcheva ${ }^{5}$
}

1Department of Gynecology, UMHAT “Saint Anna”, Varna, Bulgaria

2Department of Gynecology, Medical University Varna “Prof. Dr. Paraskev Stoyanov”, Varna, Bulgaria

${ }^{3}$ Department of General and Clinical Pathology, Forensic Medicine and Deontology, Division of General and Clinical Pathology,

Faculty of Medicine, Medical University Varna "Prof. Dr. Paraskev Stoyanov", Varna, Bulgaria

${ }^{4}$ Department of Gynecologic Oncology, Medical University Pleven, Pleven, Bulgaria

${ }^{5}$ Department of First Cardiology Clinic, UMHAT "Saint Anna”, Varna, Bulgaria

\begin{abstract}
Pseudomyxoma peritonei (PMP) is a rare and uncommon condition, characterized by the presence of mucinous ascites in the abdominal cavity. The most common cause of PMP is mucinous adenocarcinoma of the appendix, followed by neoplasms of the ovary, endocervix, fallopian tube, alimentary organs, urachus, urinary bladder, lung, mucinous cyst of the spleen, and breast.

Herein, we report a case of a 64-year-old postmenopausal woman (gravida 2, para 2) who presented at the department of gynecology with a short history of nausea and abdominal distention. Abdominal and vaginal ultrasonography showed a large amount of free fluid in the pelvis with hyperechoic echogenicity and right pelvic tumor with mixed echogenicity. Computed tomography demonstrated the presence of a heterogeneous, hypodense mass, without contrast enhancement, located on the right side of the pelvis, near the right ovary. Laparotomy was performed. Revision of the abdominal cavity revealed a large amount of yellow gelatinous mucinous ascites - approximately $1.5 \mathrm{l}$. A tumor ( $6 \times 7 \mathrm{~cm}$ in diameter), arising from the appendix and located in the pouch of Douglas near the right ovary, was observed. Histopathology examination revealed poorly differentiated mucinous appendiceal adenocarcinoma, comprising up to $50 \%$ signet ring cells.

Gastrointestinal tumors such as appendiceal neoplasms combined with PMP may mimic ovarian carcinomas. Computed tomography, abdominal/vaginal ultrasonography and tumor marker levels (carcino-embryonic antigen, carbohydrate antigen 19.9, carbohydrate antigen Ca-125) may establish the diagnosis.

A differential diagnosis with appendiceal tumors should be considered for patients with right pelvic masses.
\end{abstract}

Key words: appendiceal neoplasm, pseudomyxoma peritonei, ovarian tumors, mucin, appendectomy.

\section{Introduction}

Pseudomyxoma peritonei (PMP) is a rare and an uncommon condition, characterized by the presence of mucinous ascites in the abdominal cavity [1, 2]. The most common cause of PMP is mucinous adenocarcinoma of the appendix, followed by neoplasm of the ovary, endocervix, fallopian tube alimentary organs, urachus, urinary bladder, lung, mucinous cyst of the spleen, and breast $[3,4]$. The incidence of PMP is about 1 to 2 per million per year with a high prevalence in females (female to male ratio $2-3: 1$ ) [5]. PMP is often asymptomatic in the initial stages and the diagnosis of most patients is during surgery for suspected appendicitis, peritonitis or gynecological cancer [2, 3]. In gynecology, the premenstrual syndrome is often diagnosed during exploration for ovarian tumors [3]. The diagnosis is estimated by ultrasonography (USG), computed tomography (CT), magnetic resonance imaging (MRI) and diagnostic laparoscopy followed by histopathologic verification [5]. In gynecology, appendiceal neoplasms (AN) are often misdiagnosed as ovarian tumors [6]. Herein, a case of PMP of appendiceal origins mimicking ovarian cancer is reported.

\section{Case report}

A 64-year-old postmenopausal woman (gravida 2, para 2) presented at the department of gynecology with a short history of nausea and abdominal distention. The patient had no relevant history of urogynecological 

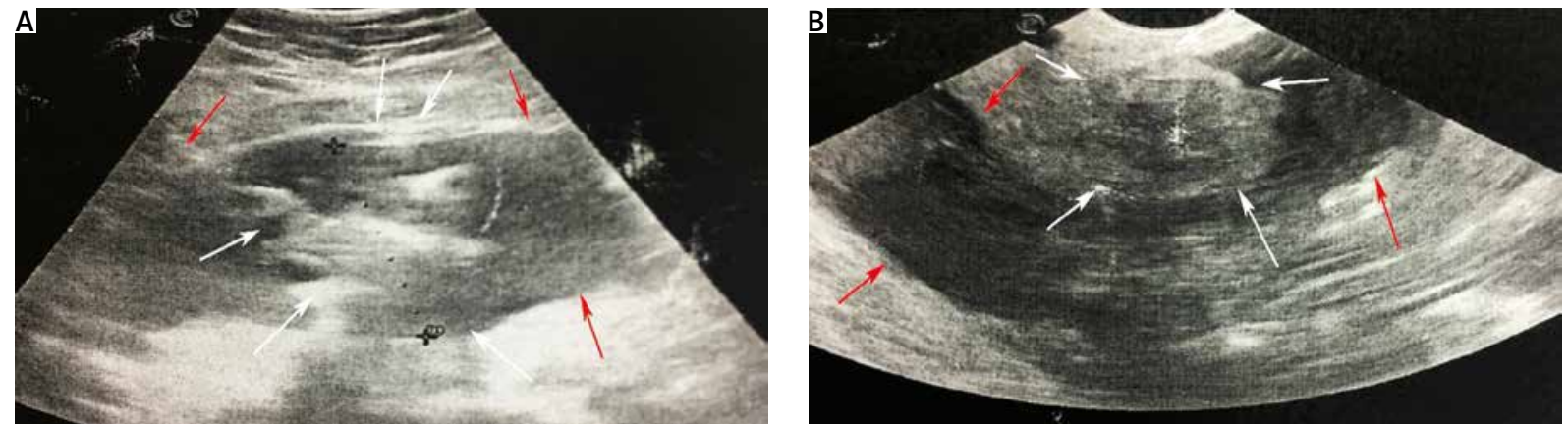

Fig. 1. Ultrasonography of the appendiceal neoplasm, mimicking right ovarian tumor. A) abdominal sonography, B) vaginal sonography. Red arrows show free fluid in the pelvis with hyperechoic echogenicity. White arrows show the tumor with mixed echogenicity
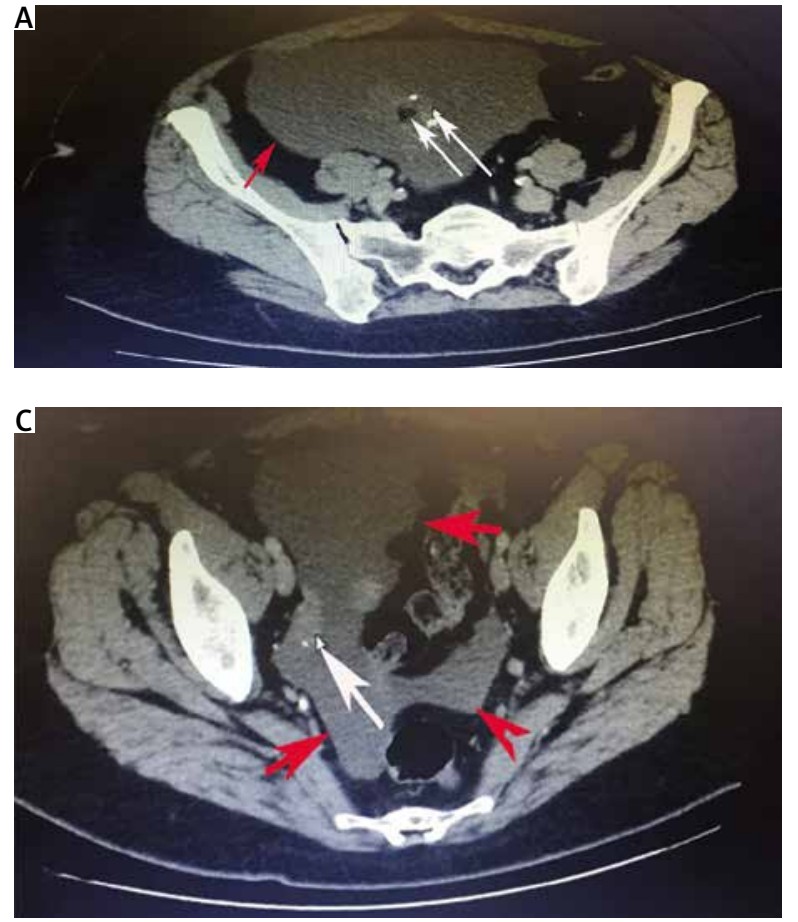

malignancies and gynecological diseases. The women had no inheritable conditions and no family cancer history in first-degree relatives. The patient had no history of tobacco use or alcohol or drug consumption. She had had arterial hypertension for 6 years. Gynecological examination revealed a palpable tender mass located near the right ovary. Abdominal and vaginal ultrasonography showed a large amount of free fluid in the abdomen with hyperechoic echogenicity and a right ovarian tumor with mixed echogenicity (Fig. 1). A laboratory examination was normal except for anemia with $10.9 \mathrm{~g} / \mathrm{dL}$ hemoglobin. The laboratory examination of tumor markers revealed elevated tumor markers - carcino-embryonic antigen (CEA) $(23.4 \mathrm{ng} / \mathrm{mL})$, carbohydrate antigen 19.9 (CA 19.9) (155.1 IU/ml) and carbohydrate antigen (CA-125) (39.4 IU/ml). Computed tomography demonstrated the presence of a heterogeneous, hypodense mass,

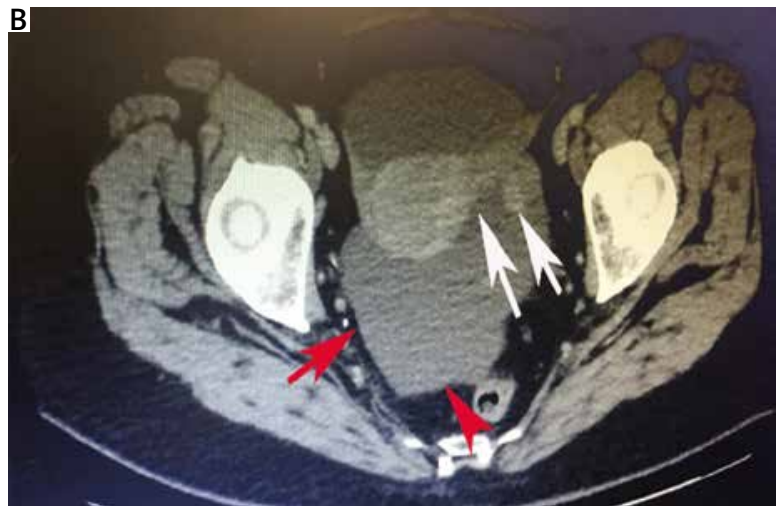

Fig. 2. Computed tomography appearance of pseudomyxoma peritonei and appendiceal neoplasm. A) a heterogeneous, hypodense mass, located on the right side of the pelvis, near the right ovary. The white arrows show the appendiceal tumor with calcification and fatty components and the lumen of the appendix. The red arrow reveals the limits of the tumor, B) the white arrows show the uterus and the left ovary. The red arrows indicate a large amount of free fluid in the peIvis, C) the white arrow points to the left ovary. The red arrows show a large amount of free fluid in the pelvis

without contrast enhancement, located on the right side of the pelvis, near the right ovary. The CT also showed central calcifications and fatty components of the tumor. Enlarged right pelvic lymph nodes and a large amount of free fluid in the abdominal cavity were also observed (Fig. 2). The patient underwent exploratory midline laparotomy, which revealed a large amount of yellow gelatinous mucinous ascites - approximately $1.5 \mathrm{l}$. A tumor (6 $\times 7 \mathrm{~cm}$ in diameter), arising from the appendix and located in the pouch of Douglas near the right ovary, was noted. Macroscopically the tumor was gross and fleshy without involvement of the base of the appendix. The right pelvic lymph nodes were enlarged. There were no implants on the parietal and visceral peritoneum. Evaluation for distant metastases was negative. Ascites fluid was obtained $(30 \mathrm{ml}$ ) for analysis. Appendectomy, total laparoscopic hysterectomy with bilateral salpingo-oo- 

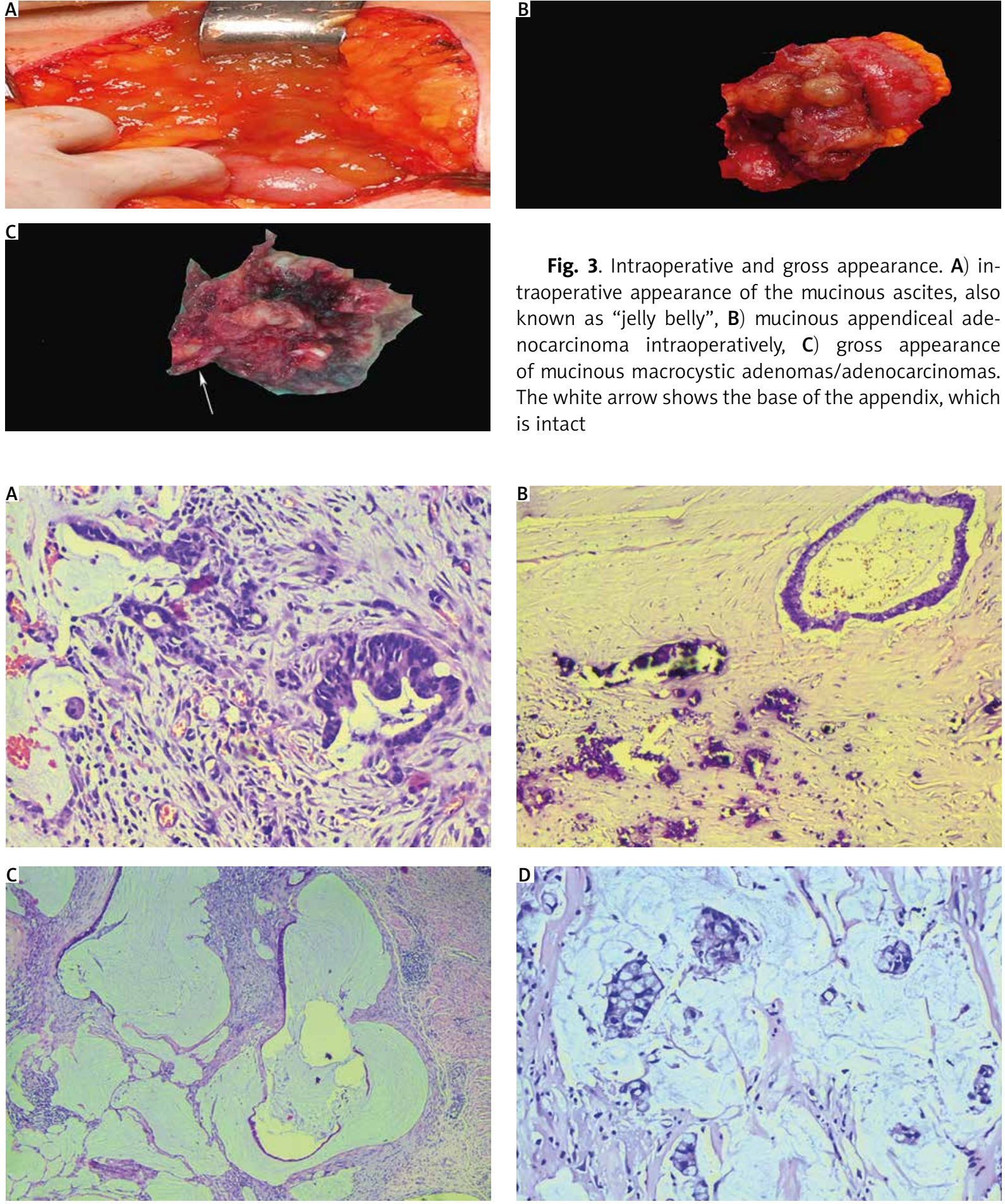

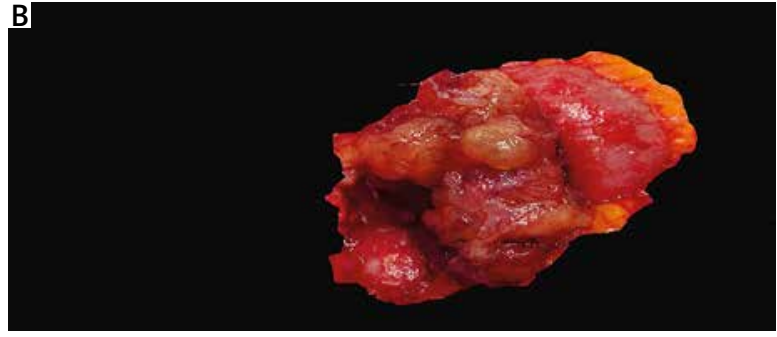

Fig. 3. Intraoperative and gross appearance. A) intraoperative appearance of the mucinous ascites, also known as "jelly belly", B) mucinous appendiceal adenocarcinoma intraoperatively, C) gross appearance of mucinous macrocystic adenomas/adenocarcinomas. The white arrow shows the base of the appendix, which is intact

Fig. 4. Microscopic appearance of the poorly differentiated mucinous adenocarcinoma with signet ring cells $(<50 \%)$. A) invasive malignant glands with desmoplastic stroma, B) malignant gland, hyalinization of stroma with dystrophic calcification, C) malignant glandular structure of colloid-filled space lines of neoplastic epithelium, D) cluster of signet ring cells floating in mucus.

A, B, D) H\&E 200x, C) H\&E 100x

phorectomy (TLHBSO) and bilateral pelvic lymph node dissection were performed. Histopathology examination revealed poorly differentiated mucinous appendiceal adenocarcinoma (MAA), comprising up to $50 \%$ signet ring cells [7]. The tumor perforated the appendix and 
TNM classification (acronym: tumor, node, metastasis) [8]. Ascites fluid analysis showed mucin without identifiable neoplastic epithelial cells. PMP was classified as acellular PMP. Intraperitoneal lavage with 5\% dextran and water was performed. We did not leave any drains in the abdominal cavity as we used enhanced recovery after surgery (ERAS) protocols [9]. The patient was discharged on the $10^{\text {th }}$ postoperative day in a good health condition. Four months after the operations, the patient is without complains. The follow-up 3 months after the operation (gynecological examination, abdominal, vaginal ultrasonography and tumor marker levels (CAE, CA-19-9, CA-125) did not show any evidence of recurrence.

\section{Discussion \\ Definition}

PMP, also known as "jelly belly," is a heterogeneous clinical disease. A comprehensive definition of PMP is still lacking $[7,10]$. Carr et al. stated that PMP could include mucinous ascites, peritoneal implants, omental cake, and ovarian involvement. It most commonly arises from appendiceal neoplasia. Authors have concluded that PMP represents a macroscopic appearance of mucinous ascites in the abdominal cavity and it is not a histologic diagnosis [7, 10].

\section{Origin}

As the second most common cause of PMP in women is ovarian MAA, it was believed that PMP commonly originated from ovarian malignancies. This theory has now been refuted. Currently, immuno-histochemistry and molecular genetic examinations support the theory that ovarian mucinous adenocarcinomas are metastases from a perforated appendiceal mucinous tumor [2, 3]. Women presented at an earlier stage than men [2].

\section{Etiology}

Patients with familial adenomatous polyposis and KRAS, GNAS, TP53 mutations have an elevated risk of developing mucinous adenocarcinoma of the appendix. KRAS mutation is distributed among both low and high grade PMP. It has been observed in $70 \%$ of patients with appendiceal adenomas [11-13]. GNAS mutation is more common in low grade than high grade PMP, whereas TP53 mutations are prevalent in high grade PMP [13]. TP53 overexpression is associated with female sex, high grade PMP and poor outcome [13].

\section{Epidemiology}

As mentioned above, according to some authors the estimated incidence of PMP is about 1 to 2 per million per year, whereas other authors reported incidence of PMP approximately 1 to 4 per million per year $[5,11]$.

\section{Pathophysiology}

The pathophysiology of PMP is explained in Figure 5 $[2,3,13,14]$.

\section{Pathology}

There are differences and controversies over the classification of epithelial appendiceal neoplasms and its relation to PMP. It is necessary to describe and

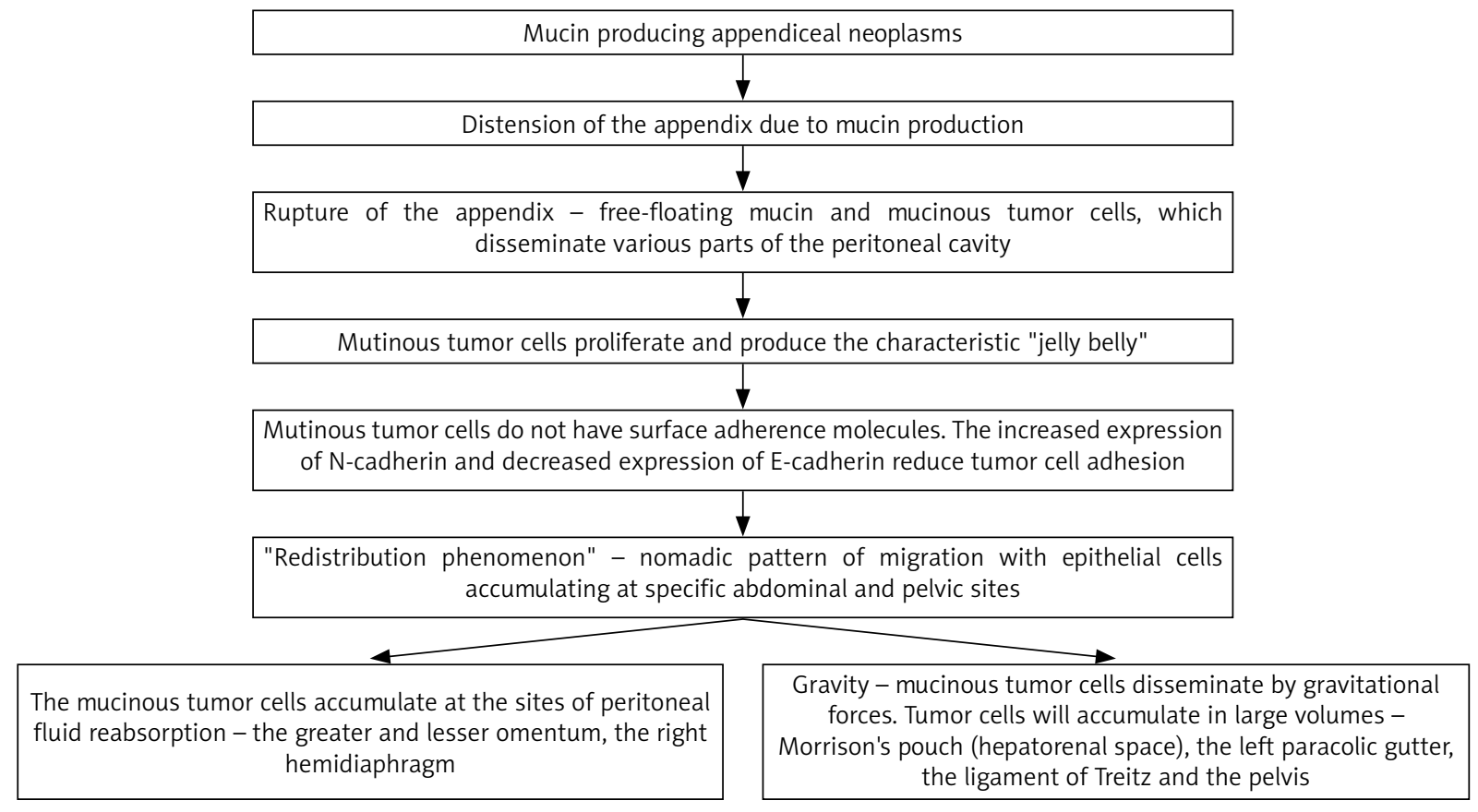

Fig. 5. Pathophysiology of pseudomyxoma peritonei 


\begin{tabular}{|c|c|}
\hline Diagnostic terminology & Histological characteristic \\
\hline Acellular PMP & $\begin{array}{l}\text { - Mucin without neoplastic epithelial cells, confined to vicinity of the organ of origin or distant } \\
\text { from it }\end{array}$ \\
\hline \multirow[t]{4}{*}{ LAMN PMP } & - Low-grade cytologic atypia \\
\hline & - Epithelial component typically scanty (<20\% tumor volume) \\
\hline & - Few mitoses \\
\hline & - Invasion into underlying organs is generally of the "pushing" type \\
\hline \multirow[t]{5}{*}{ HAMN PMP } & - High-grade cytologic atypia \\
\hline & - Infiltrative invasion into adjacent tissue \\
\hline & - Angiolymphatic or perineural invasion \\
\hline & - Cribriform growth \\
\hline & - Numerous mitoses \\
\hline HAMN PMP with signet ring cells & - Neoplasm with signet ring cell component (minimum 10\% signet ring cells) \\
\hline
\end{tabular}

Fig. 6. Classification of pseudomyxoma peritonei $[7,10,16]$

subdivide the histologic grade of the peritoneal disease and the primary tumor, as both having an individual histological subclassification, sometimes with discordance. For instance, in the present case, the patient had poorly differentiated MAA with signet ring cells and acellular PMP. Other authors have described such a discordance - patients with low-grade appendiceal mucinous neoplasm (LAMN) may present with high-grade PMP, whereas high-grade appendiceal mucinous neoplasm (HAMN) could cause low-grade PMP [3, 7, 10]. Mucinous appendiceal neoplasms which can be associated with PMP include LAMN, HAMN, MAA (well/ moderately/poorly differentiated), poorly differentiated MAA with signet ring cells ( $<50 \%$ signet ring cells), mucinous signet ring cell adenocarcinoma ( $>50 \%$ signet ring cells) and goblet cell carcinoids [7, 10, 13, 15]. Classification of PMP is shown in Figure $6[7,10,16]$.

\section{Prognostic factors}

Prognostic factors include the following - histological grade of primary tumor and PMP, the stage of local invasion throughout the peritoneal cavity, presence/ absence of neoplastic cells on the appendiceal serosa, presence/absence of signet ring cells, resection margins status, tumor marker levels (CAE, CA-125, CA-19-9), neutrophil-lymphocyte ratio, perforated/non-perforated appendicitis, completeness of cytoreduction, peritoneal cancer index, lymph node involvement, TP53 mutations, initiation of hyperthermic intraperitoneal chemotherapy (HIPEC) [1-3, 10, 13].

\section{Clinical features}

Clinicalfeaturesconsistofunspecifiedsignsand symptoms that correlate with the progression of the disease - abdominal girth, an appendicitis-like syndrome, irritable bowel syndrome, new-onset hernia. An advanced disease may result in ascites, bowel obstruction, malnutrition, and palpable abdominal masses (omental cake, ovarian masses in women) $[2,3,11]$.

\section{Diagnosis}

Imaging modalities, mainly ultrasound and $\mathrm{CT}$, may establish the diagnosis $[2,5,11,17]$. A specific sonography marker is the so-called "onion skin sign" and its "dumbbell structure" [18]. Typical CT appearance is 'scalloping' of the surface of the liver and spleen, which distinguishes mucinous from fluid ascites $[1,11]$. A computed tomography scan can also show low-attenuation lesions or loculated ascites scattered throughout the peritoneal cavity and individual peritoneal or omental nodules (omental cake in advance disease). Often it is hard to detect the primary tumor in a CT scan [17]. Moreover, many authors have reported appendiceal tumors and PMP, which mimic ovarian masses, and the patient underwent surgery with this diagnosis, as in our case [18-20]. Demir et al. reported a case of a patient who underwent an operation for a suspected right ovarian tumor. Intraoperatively, they found a $6 \times 4 \mathrm{~cm}$ cystic mass that originated from the appendix. Genital organs were normal. Histopathology revealed mucocele of the appendix. The authors concluded that appendiceal neoplasms might be considered as a differential diagnosis in cases of right pelvic tumors [18]. Akman et al. reported a case of a large mucinous neoplasm of the appendix mimicking an adnexal mass in a postmenopausal woman. The authors stated that in such cases, the preoperative diagnosis remains difficult [18]. Pantiora et al. concluded that it is essential for gynecologists and general surgeons to consider the presence of AN in the case of an elderly woman with a tumor located in the right iliac fossa [19].

The significance of serum tumor marker levels (CAE, CA-19-9, CA-125) includes diagnosis, prognosis, predictors of incomplete cytoreduction, follow-up and early identification of recurrence [2].

\section{Treatment}

Currently, the treatment regimens include optimal cytoreductive surgery (OCS) and HIPEC [6]. HIPEC is 
used at $42^{\circ} \mathrm{C}$, and the chemotherapeutic agents used are mitomycin C, 5-fluorouracil, cisplatin, and oxaliplatin. Mitomycin C is most commonly used among patients with PMP. OCS includes a complex series of peritonectomies performed in a single procedure. OCS is considered optimal if no macroscopic residual disease is visible [2]. It was reported that in advanced disease HIPEC did not impact the overall survival of patients, whereas OCS did [2]. We performed TLHBSO, as in postmenopausal women with MAA, the performance of bilateral salpingo-oophorectomy, regardless of the macroscopic appearance of the ovaries, reduces recurrence and avoids second primaries. Moreover, ovarian metastases are commonly metachronous [10]. As it is difficult to differentiate HAMN and LAMN in frozen section, we performed only appendectomy. It is stated that in cases of uncertainty or an unexpected finding of PMP during the course of elective abdominal surgery, it may be preferable to perform an appendectomy and wait for the definitive histopathology [2, 20]. Right hemicolectomy $(\mathrm{RH})$ could be performed initially or as a second operation after multidisciplinary team consultation $[2,6,10,20]$. Right hemicolectomy is performed in patients with high-grade appendiceal histology with or without PMP. In perforated appendices, or clinical signs of PMP, RH should be considered if it is part of a complete CRS and HIPEC. For patients with MAA and PMP, the follow-up includes physical examination, thoracic/abdominal/pelvic CT scan and tumor marker levels (CAE, CA-19-9, CA-125). Govaerts et al. concluded that in patients with HAMN (defined after histopathology) and any $\mathrm{pT}, \mathrm{pNx}, \mathrm{pM} 1 \mathrm{~b}, \mathrm{RO} \mathrm{N}^{* *}$ and no postoperative radiological/biochemical signs of residual disease, a CRS and HIPEC with a right sided hemicolectomy should or could be performed [10].

\section{Conclusions}

Appendiceal mucinous neoplasms combined with PMP are rare entities, which could mimic ovarian tumors. A differential diagnosis with appendiceal tumors should be considered for patients with right pelvic masses.

\section{Acknowledgements}

Written informed consent was obtained from the patient for publication of this case report and accompanying images.

This is a case report and it did not require ethical approval from an ethics committee according to our institution.

\section{Disclosure}

The authors report no conflict of interest.

\section{References}

1. García KM, Flores KM, Ruiz A, González FL, Rodríguez ÁM. Pseudomyxoma peritonei: case report and literature review. J Gastrointest Cancer 2019; 50: 1037-1042.

2. Mittal R, Chandramohan A, Moran B. Pseudomyxoma peritonei: natural history and treatment. Int J Hyperther 2017; 33: 511-519.

3. Bevan KE, Mohamed F, Moran BJ. Pseudomyxoma peritonei. World J Gastrointest Oncol 2010; 2: 44-50.

4. Agrawal AK, Bobiński P, Grzebieniak Z, et al. Pseudomyxoma peritonei originating from urachus-case report and review of the literature. Curr Oncol 2014; 21: e155-e165.

5. Ioannidis O, Cheva A, Paraskevas G, et al. Pseudomyxoma retroperitonei: report of 2 cases and review of the literature. Rev Esp Enferm Dig 2012; 104: 268-275.

6. Demir M, Çetinkaya Z, Tavusbay C, Şengul M, Kiziltuğ N, Yilmaz B. Mucocele of appendix mimicking ovarian malignancy in a postmenopausal woman: report of a case. Available at: https://www.researchgate.net/ publication/322504864_Mucocele_of_appendix_mimicking_ovarian_ malignancy_in_a_postmenopausal_woman_report_of_a_case.

7. Legué LM, Creemers G-J, de Hingh IHJT, Lemmens VEPP, Huysentruyt CJ. Review: pathology and its clinical relevance of mucinous appendiceal neoplasms and pseudomyxoma peritonei. Clin Colorectal Cancer 2018; 18: 1-7.

8. Gonzalez RS. Staging-carcinoma. PathologyOutlines.com website. Available at: https://www.pathologyoutlines.com/topic/appendixstaging. html.

9. Melnyk M, Casey RG, Black P, Koupparis AJ. Enhanced recovery after surgery (ERAS) protocols: time to change practice? Can Urol Assoc J 2011; 5: 342-348.

10. Govaerts K, Lurvink RJ, de Hingh IHJT, et al. Appendiceal tumours and pseudomyxoma peritonei: literature review with PSOGI/EURACAN Clinical Practice Guidelines for diagnosis and treatment. Eur J Surg Oncol 2021; 47: 11-35.

11. Carr NJ, Cecil TD, Mohamed F, et al. A consensus for classification and pathologic reporting of pseudomyxoma peritonei and associated appendiceal neoplasia. Am J Surg Pathol 2016; 40: 14-26.

12. Shih IM, Yan H, Speyrer D, Shmookler BM, Sugarbaker PH, Ronnett BM. Molecular genetic analysis of appendiceal mucinous adenomas in identical twins, including one with pseudomyxoma peritonei. Am J Surg Pathol 2001; 25: 1095-1099.

13. Bignell M, Carr NJ, Mohamed F. Pathophysiology and classification of pseudomyxoma peritonei. Pleura Peritoneum 2016; 1: 3-13.

14. Sugarbaker PH. Pseudomyxoma peritonei. A cancer whose biology is characterised by a redistribution phenomenon. Ann Surg 1994; 219: 109-111.

15. Yu B, Raj MS. Pseudomyxoma Peritonei. Available at: https://www.ncbi. nlm.nih.gov/books/NBK541116/.

16. Carr NJ, Bibeau F, Bradley RF. The histopathological classification, diagnosis and differential diagnosis of mucinous appendiceal neoplasms, appendiceal adenocarcinomas and pseudomyxoma peritonei. Histopathol 2017; 71: 847-858.

17. Fonseca C, Carvalho S, Cunha TM, Gil RT, Abecasis N. The many faces of pseudomyxoma peritonei: a radiological review based on 30 cases. Radiol Bras 2019; 52: 372-377.

18. Akman L, Hursitoglu BS, Hortu i, Sezer T, Oztekin K, Avsargil BD. Large mucinous neoplasm of the appendix mimicking adnexal mass in a postmenopausal woman. Int J Surg Case Rep 2014: 5: 1265-1267.

19. Pantiora EV, Massaras D, Koutalas J, Bagiasta A, Kontis EA, Fragulidis GP. Low-grade appendiceal mucinous neoplasm presenting as adnexal mass: a case report. Cureus 2018; 10: e3568.

20. Murphy EMA, Farquharson SM, Moran BJ. Management of an unexpected appendiceal neoplasm. Br J Surg 2006; 93: 783-792. 